\title{
Two-Layered Hierarchical Spectrum Sharing in Cooperative Cognitive Radio Networks: A Stable Matching with Peer Effects Approach
}

\author{
Ting-Chen Chen", Min-Kuan Chang ${ }^{\mathrm{a},{ }^{*}}$, Feng-Tsun Chien ${ }^{\mathrm{b}}$, Yu-Wei Chan ${ }^{\mathrm{c}}$ \\ ${ }^{a}$ Graduate Institute of Communication, National Chung Hsing University, Taiwan \\ *Department of Electrical Engineering, National Chung Hsing University, Taiwan \\ ${ }^{b}$ Department of Electronics Engineering, National Chiao Tung University, Taiwan \\ 'Department of Information Management, Chung Chou University of Science and Technology, Taiwan \\ Email: ${ }^{a}$ minkuanc@dragon.nchu.edu.tw, ${ }^{b} \mathrm{ftchien@mail.nctu.edu.tw,}{ }^{\mathrm{c} y w} \mathrm{w}$ chan@dragon.ccut.edu.tw
}

\begin{abstract}
Abstract - In this paper, we mainly address the problem of two-layered spectrum sharing between primary users (PUs) and secondary users (SUs) in a multi-channel cooperative cognitive radio network (CCRN). In CCRN, PUs select SUs as relays to increase their transmission rates while SUs gain the spectrum usage opportunities for transmitting their own traffics. However, how to select appropriate SUs as relays for PUs is still an important research issue, particularly in such an environment of multi-channel with multiple PUs and multiple SUs. In this work, we propose an approach of stable matching with peer effects, in which the stable matching and the swap matching algorithms are proposed to form the matched pairings between PUs and SUs, such that the SU will relay its paired-PU's data in exchange for gaining the spectrum usage opportunities. Finally, the benefits of the stable matching and the swap matching approaches comparing to the ones of random matching and optimal allocation are demonstrated by the simulations.
\end{abstract}

Keywords: Cooperative cognitive radio networks, stable matching, peer effects, hierarchical spectrum sharing.

\section{Introduction}

In recent years, cooperative cognitive radio networks $(\mathrm{CCRN})^{(1-4)}$ has emerged as an efficient and power-saving communication paradigm, in which the SUs act as cooperative relays to assist the PUs' transmission in exchange for accessing the spectrum opportunities. Thus, a "win-win" situation can be achieved where the PUs can increase their transmission rates while the SUs can gain the spectrum usage opportunities for transmitting their own traffics. In CCRN, PUs can achieve larger transmission rates by selecting the SUs as their relays than by using the direct transmission. However, it is challenging for PUs to select appropriate SUs as relays, particularly in an environment of multi-channel with multiple PUs and multiple SUs. In addition, the consideration of one channel and one PU in previous works $^{(1,2)}$ presents a simplification for practical scenarios where there are typically multiple channels and multiple PUs that coexist in the coverage area of a base station in the cellular network.

In this paper, we investigate the cooperative spectrum sharing strategies between multiple PUs and multiple SUs in a multi-channel CCRN. We apply the stable matching theory ${ }^{(5-8)}$ to determine the cooperative relays for PUs in a two-layered hierarchical spectrum sharing environment.

Stable matching theory proposed by Gale-Shapley ${ }^{(5)}$ has been applied recently to solve the resource allocation problems in wireless networks ${ }^{(8-11)}$. The basic concept of stable matching theory is to introduce how the players form stable matched pairings with their preferences ${ }^{(6,7,9)}$. In (10), a distributed matching algorithm was proposed to investigate the spectrum sharing strategies between multiple PUs and multiple SUs in a cognitive radio network. Thus, the matched pairings between PUs and SUs are determined, such that the SUs will relay their paired PUs' data, in exchange for spectrum access.

In this paper, we first propose a novel two-layered 
hierarchical spectrum sharing framework between multiple PUs and multiple SUs. In the first layer, a cooperative spectrum sharing model between PUs and SUs is proposed. At the same time, the matched pairings between them are determined by the proposed stable matching algorithms. In the second layer, the cooperation model among SUs is proposed, in which the SUs who use the subchannel leased from the same PU will form a cooperative group. In the group, one SU can select other SUs as its relays for achieving higher capacities. However, the problem of peer effects among SUs may exist due to their underlying social relations (or networks) ${ }^{(12)}$, such that the stable matching results between PUs and SUs obtained from the first layer will be affected by peer effects. Thus, we then propose the swap matching algorithm to solve the peer effects problem. Finally, the benefits of the stable matching and the swap matching approaches comparing to the ones of random matching and optimal allocation are verified by the simulation results.

\section{SYSTEM MODEL}

\subsection{System Setup}

We consider the uplink transmission of a single cell FDMA-based cognitive radio network coexisted with a primary licensed network, comprising of $N_{p}$ PUs, a primary receiver called the primary base station (PBS), $N_{s}$ SUs, and a cognitive receiver called the secondary access point (SAP). Let the set of PUs and SUs be $N_{p}=\left\{1, \ldots, N_{p}\right\}$ and $N_{s}=$ $\left\{1, \ldots, N_{s}\right\}$, respectively. We define that a $\mathrm{PU}$ and a $\mathrm{SU}$ are matched if the SU cooperatively relays the PU's data, in exchange for spectrum access. The formed matching pair is denoted by $\left(P U_{i}, S U_{j}\right)$, for $1 \leq i \leq N_{p}$ and $1 \leq i \leq N_{s}$. Each PU attempts to grant spectrum access to a $\mathrm{SU}$, as determined by the various matching algorithms, in exchange for that the SU cooperatively relays the PU's data to the destination PBS. For simplicity, we make the following assumptions regarding the cooperative transmission model:

1) We assume that each PU has only one available subchannel which can be leased to the matched SUs, but the PU can select only one SU every time. In addition, we assume that one subchannel can be leased to two SUs simultaneously. Moreover, we also assume that each PU has $q_{p u}$ quotas for providing SUs opportunities of spectrum access. This means that each PU can select at most $q_{p u}$ SUs as its relay nodes.

2) The set of all PUs' subchannels in the primary network is assumed to be $S_{m}=\left\{1, \ldots, N_{p}\right\}$.
3) We assume that each SU experiences different channel conditions of all paths when they are using channels leased by different PUs.

4) Amplify-and-forward (AF) protocol is adopted at SUs to relay PUs' traffic, and equal power allocation is employed for all PUs and SUs. Besides, we assume that flat Rayleigh faded channel for each link that is invariant within each slot. At the PBS, the receiver employs maximal ratio combining (MRC) to combine signals from the direct link and the relay links. The channel state information (CSI) is assumed available at the PBS.

In this work, we propose a novel hierarchical spectrum sharing framework, in which there are two layers. In the first layer, each PU selects multiple SUs as relay nodes for transmitting its own traffics while the selected SUs gain opportunities for spectrum access. We assume that each PU will select the same number of SUs, which is denoted by $\delta_{q}$, $\delta_{q}=\left\{2, \ldots, \delta_{q}\right\}$, to form the matched pairs. For instance, the formed matching pairings between two PUs and four SUs are exemplified as shown in Fig. 1. In the second layer, the cooperation model among SUs is proposed, which is shown in Fig. 2. In this layer, SUs which use the same subchannel leased from one of the PUs form a cooperative group, in which one SU can select other SUs as its relay nodes for achieving higher capacities. In addition, the peer effects among SUs are considered, so that SUs will exchange their matched PUs for achieving higher capacities.

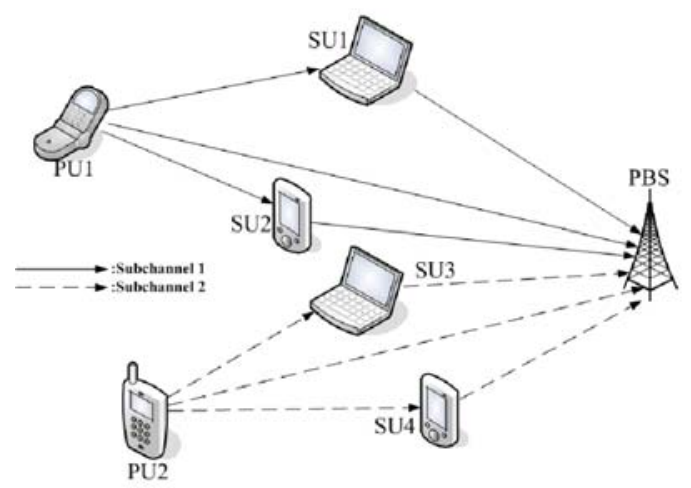

Figure 1 The matched pairings formed between PUs and 


\section{SUs}

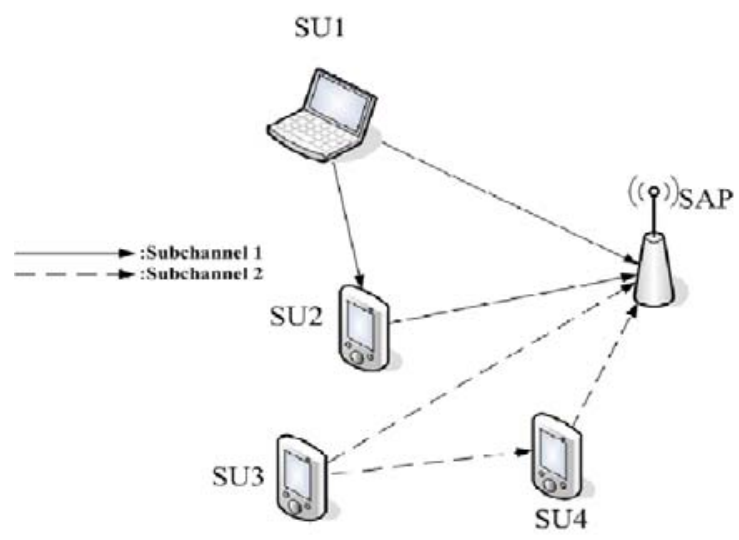

Figure 2 The SUs' cooperation model

\subsection{Utility Functions of PUs and SUs}

With the cooperation mechanism described in the previous section, we are now ready to define each player's utility based on their respective achievable capacity. First, the achievable capacity of PU $i$ with the help of SU $j$ is given by the following formula:

$$
\begin{aligned}
\mathrm{C}_{P U_{i}, S U_{j}}^{(\mathrm{m})}=\log _{2}(1 & +\frac{\gamma_{P U}\left|h_{P U_{i}, P B S}^{(m)}\right|^{2}}{d_{P U_{i}, P B S}^{\alpha}} \\
+ & \frac{\frac{\gamma_{P U} \gamma_{S U}\left|h_{P U_{i}, S U_{j}}^{(m)}\right|^{2}\left|h_{S U_{j}, P B S}^{(m)}\right|^{2}}{d_{P U_{i}, S U_{j}}^{\alpha} d_{S U_{j}, P B S}^{\alpha}}}{\frac{\gamma_{P U}\left|h_{P U_{i}, S U_{j}}^{(m)}\right|^{2}}{d_{P U_{i}, S U_{j}}^{\alpha}}+\frac{\gamma_{S U}\left|h_{S U_{j}, P B S}^{(m)}\right|^{2}}{d_{S U_{j}, P B S}^{\alpha}}+1}
\end{aligned}
$$

where $\gamma_{\mathrm{SU}_{\mathrm{j}}}=\frac{P_{P U_{j}}}{\sigma^{2}}$ and $\gamma_{\mathrm{SU}_{\mathrm{k}}}=\frac{P_{S U_{k}}}{\sigma^{2}}, \sigma^{2}$ is the noise variance, and $\alpha$ is the path loss. $P_{P U_{i}}$ and $P_{S U_{j}}$ are the transmission power of $\mathrm{PU} i$ and $\mathrm{SU} j$, respectively. In particular, $m$ represents the $m^{\text {th }}$ subchannel which is leased to $\mathrm{SU} j$ by PU $i$. The notations of $d_{P U_{i}, S U_{j}}$ and $d_{S U_{i}, P B S}$ represent the distances from PU $i$ to $\mathrm{SU} j$ and the ones from SU $j$ to the PBS, respectively.

In addition, we use the following notations to denote the channel gain between different transmitter-receiver pairs: $h_{P U_{i}, P B S}^{(m)}$ denotes the channel gain of the subchannel $m$ from PU $i$ to the PBS; $h_{P U_{i}, S U_{j}}^{(m)}$ denotes the channel gain of the subchannel $m$ between $\mathrm{PU} i$ and $\mathrm{SU} j ; h_{S U}^{(m)}(P B S$ denotes the channel gain of the subchannel $m$ from $\mathrm{SU} j$ to the PBS.

Second, the capacity of the direct transmission from PU $i$ to the PBS is given by

$$
C_{P U_{i}, P B S}^{(d i r e c t)}=\log _{2}\left(1+\frac{\gamma_{P U}\left|h_{P U_{i}, P B S}^{(m)}\right|^{2}}{d_{P U_{i}, P B S}^{\alpha}}\right),
$$

where $h_{P U_{i}, P B S}^{(m)}$ denotes the channel gain of the subchannel $m$ from PU $i$ to the PBS. In addition, $d_{P U_{i}, P B S}$ denotes the distances between PU $i$ and the PBS.

Next, let us look at the utility function of SUs. First, in the direct transmission, since the SUs who act as cognitive relays have opportunities to use the available subchannels for transmitting their traffics, the capacity of SU $j$ is given by

$$
C_{S U_{j}, S A P}^{(\text {direct })}=\log _{2}\left(1+\frac{\gamma_{S U}\left|h_{S_{j}, S A P}^{(m)}\right|^{2}}{d_{S U_{j}, S A P}^{\alpha}}\right),
$$

where $h_{S U_{j}, S A P}^{(m)}$ and $d_{S U_{j}, S A P}$ denote the channel gain of the subchannel $\$ \mathrm{~m} \$$ from SU $j$ to the SAP and the distances from $S U_{j}$ to the SAP, respectively.

Second, due to the proposed cooperation model among SUs, the SU will select other SUs who are using the same subchannel as relay nodes. Thus, the capacity of $\mathrm{SU} j$ achieved from the $\mathrm{SU} j$ to the PBS with the relay helped by $\mathrm{SU} k$ is represented by

$$
\begin{aligned}
& \mathrm{C}_{S U_{j}, k, S A P}^{(\mathrm{m})} \\
& =\log _{2}\left(1+\frac{\gamma_{S U_{j}}\left|h_{S U_{j}, S A P}^{(m)}\right|^{2}}{d_{S U_{j}, S A P}^{\alpha}}\right. \\
& \left.+\frac{\frac{\gamma_{S U_{j}} \gamma_{S U_{k}}\left|h_{S U_{j}, S U_{k}}^{(m)}\right|^{2}\left|h_{S U_{k}, S A P}^{(m)}\right|^{2}}{d_{S U_{j}, S U_{k}}^{\alpha} d_{S U_{k}, S A P}^{\alpha}}}{\frac{\gamma_{S U_{j}}\left|h_{S U_{j}, S U_{k}}^{(m)}\right|^{2}}{d_{S U_{j}, S U_{k}}^{\alpha}}+\frac{\gamma_{S U}\left|h_{S U_{k}, S A P}^{(m)}\right|^{2}}{d_{S U_{k}, S A P}^{\alpha}}+1}\right)
\end{aligned}
$$

where $\quad \gamma_{S U_{j}}=\frac{P_{S U_{j}}}{\sigma^{2}}$ and $\gamma_{S U_{k}}=\frac{P_{S U_{k}}}{\sigma^{2}}, P_{S U_{j}}$ and $P_{S U_{k}}$ denote the transmission power of $S U_{j}$ and $S U_{k}$, respectively. In addition, $h_{S U_{j} S U_{k}}$ and $h_{S U_{j} S A P}$ are the channel gain between $S U_{j}$ and $S U_{k}$ and from $S U_{k}$ to the SAP, respectively. In addition, $d_{S U_{j}, S U_{k}}$ and $d_{S U_{j}, S A P}$ denote the distances between $S U_{j}$ and $S U_{k}$ and the ones from $S U_{k}$ to the SAP, respectively. 
Finally, the PBS combines the signals of two SUs by employing the maximal ratio combining (MRC) to calculate the capacity achieved from SU $j$ to the PBS, which is

$\mathrm{C}_{S U_{j}, k, P B S}^{(m)}=\log _{2}\left(1+\frac{\gamma_{P U}\left|h_{P U_{i}, P B S}^{(m)}\right|^{2}}{d_{P U_{i}, P B S}^{\alpha}}+\right.$

$\frac{\frac{\left.\gamma_{P U} \gamma_{S U}\left|h_{P U_{i}, S U_{j}}^{(m)}\right|\right|^{2}\left|h_{S U_{j}, P B S}^{(m)}\right|^{2}}{d_{P U_{i}, S U_{j}}^{\alpha} d_{S U_{j}, P B S}^{\alpha}}}{\frac{\gamma_{P U}\left|h_{P U_{i}, S U_{j}}^{(m)}\right|^{2}}{d_{P U_{i}, S U_{j}}^{\alpha}}+\frac{\gamma_{S U}\left|h_{S U_{j}, P B S}^{(m)}\right|^{2}}{d_{S U_{i}, P B S}^{\alpha}}+1}+$

$\left.\frac{\frac{\gamma_{P U} \gamma_{S U}\left|h_{P U_{i}, S U_{k}}^{(m)}\right|^{2}\left|h_{S U_{k}, P B S}^{(m)}\right|^{2}}{d_{P U_{i}, S U_{k}}^{\alpha} d_{S U_{k}, P B S}^{\alpha}}}{\frac{\gamma_{P U}\left|h_{P U_{i}, S U_{k}}^{(m)}\right|^{2}}{d_{P U_{i}, S U_{k}}^{\alpha}}+\frac{\gamma_{S U}\left|h_{S U_{k}, P B S}^{(m)}\right|^{2}}{d_{S U_{k}, P B S}^{\alpha}}+1}\right)$

Note that PUs and SUs are selfish entities interested in maximizing their own utility. They will have incentives to form the matched pairings only when this will increase their own individual utility besides the aggregate utility. In the following section, we will adopt the stable matching theory to analyze the behavior of the proposed cooperative network.

\section{STABLE MATCHING THEORETIC FORMULATION}

\subsection{Preference Lists of PUs and SUs}

Each PU has a preference list of SUs which is constructed by the following criterion:

$$
C_{P U_{i}, S U_{j}}^{(m)}>C_{P U_{i}, S U_{k}}^{(m)}
$$

In (6), if the PU $i^{\prime} s$ achieved capacity with the $\mathrm{SU} j$ as the relay node is greater than the one with the $\mathrm{SU} k$, then the $\mathrm{SU}$ $j$ will be selected as one of the candidate matching partner in the preference list of PU $i$.

Similarly, each SU has a preference list of PUs which is formed mainly by the following criterion:

$$
C_{S U_{j}, S A P}^{(m)}>C_{S U_{i, S A P}}^{(n)},
$$

where the notation $C_{S U_{j}, S A P}^{(m)}$ means that $S U j^{\prime} s$ capacity is achieved by accessing PU $m^{\prime} s$ subchannel. If the achieved capacity by using the subchannel of PU $m$ is greater than the one by using the subchannel of PU $n$, then the PU $m$ will be selected as the candidate partner in SU $j^{\prime} s$ preference list.

\subsection{The Proposed Algorithms}

Next, the stable matching algorithm and the swap matching algorithm will be proposed, which are introduced as follows:

\subsubsection{The Stable Matching Algorithm}

The algorithm is proposed to determine the matched pairings between PUs and SUs, such that the SU will relay its paired-PU's data in exchange for spectrum access. The matching process is given by Algorithm 1. In Algorithm 1, we first assume that each PU has two quotas of subchannel which can be leased to SUs. Each PU will send an offer request to the $\mathrm{SU}$ which is first in its preference list. This $\mathrm{SU}$ will either (1) accept the offer if the PU is in the SU's preference list, in which case the PU and SU are matched, or (2) reject the offer if the PU is not in the SU's preference list. The process details of the algorithm are as follows:

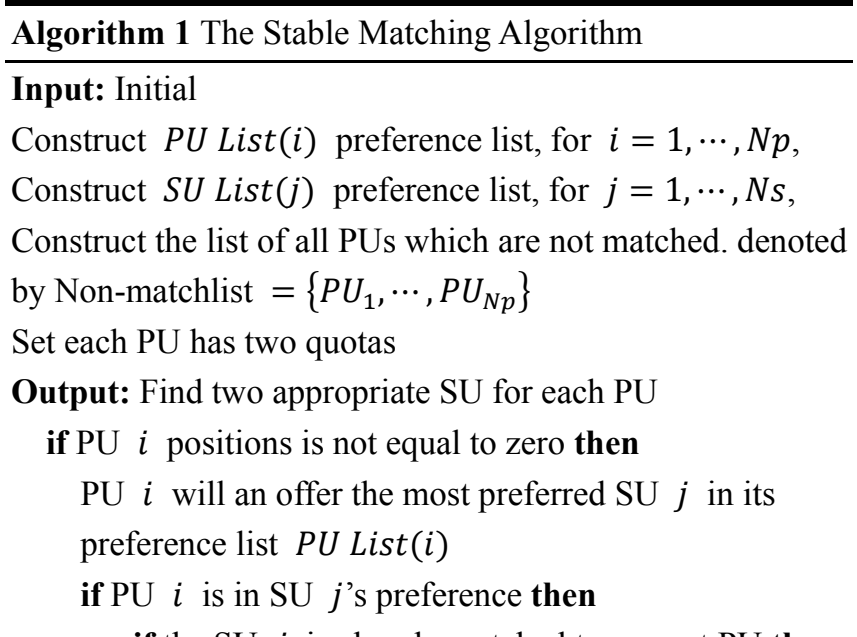

if the $\mathrm{SU} j$ is already matched to current PU then

if the PU $i$ 's capacity is better than current PU on SU $j$ 's preference list $S U \operatorname{List}(j)$ then

Remove current PU match

$\mathrm{PU} i$ and $\mathrm{SU} \mathrm{j}$ are matched

PU $i$ positions minus one and the current

$\mathrm{PU}$ positions add one

end if

else

$\mathrm{PU} i$ and $\mathrm{SU} \mathrm{j}$ are matched

The number of PU $i$ 's quotas minus one end if

\section{end if}

If the PU $i$ has no positions, then remove PU $i$ from Non-matchlist

end if

If all $P U s$ has no positions, then stop the algorithm. Else go to start for each PU which positions is not equal to zero 


\subsubsection{The Swap Matching Algorithm:}

The stable matching pairings between PUs and SUs are formed in the first phase, via the processes presented in Algorithm 1. Next, let's see the cooperation strategy among SUs in the second phase. In this phase, SUs which use the same subchannel form a cooperative group, in which one SU select other SUs as relay nodes for increasing capacity. However, due to the underlying social connections among SUs, some SUs may propose the swap requests of using other subchannels to other SUs. For instance, SUs may be good friends, such that they want to use the same subchannel. Thus, peer effects may occur during the SUs' cooperation period.

We discuss the two-sided swap matching approaches. In the two-sided swap matching, both the capacities of PUs and SUs should be simultaneously considered when the SUs decide whether to swap or not. This means that if the achieved capacities after swapping for both PUs and SUs are larger than the ones of their respective current matching, then the SUs will swap. The algorithms of the two-sided swap matching are presented in Algorithm 2.

\section{Algorithm 2 The Two-Sided Swap Matching Algorithm}

\section{Input: Initial}

Construct the SU propose list and using channel, denoted by $S U p r o o_{j}^{i}$, for $i=1, \ldots, N p$, and $j=1, \ldots, N s$

Output: Find appropriate swap SU

if after both $S U p r o_{j}^{i}$ and $S U_{j}^{i}$ are swapping that capacity better then current matching in different channel, and other matching capacity can't getting worse then

$$
S U \operatorname{pro}_{j}^{i} \text { and } S U_{j}^{i} \text { are swap matching }
$$

else

$$
\begin{aligned}
& S U \operatorname{Sro}_{j}^{i} \text { and } S U_{j}^{i} \text { are not swapping } \\
& \text { end if }
\end{aligned}
$$

If no SU can find other PU's channel can better than current matching, then stop the algorithm

\section{SIMULATION RESULTS}

In this section, we simulate one scenario, in which each PU has two quotas which can be offered to SUs when forming the matched pairings. This means that one PU can be matched with two SUs sequentially.

We assume that the number of subchannel of each PU for lease is assumed to be one for simplifying the protocol design. The PBS and the PUs are assumed to be located on a squared network of length four, and the SAP and the SUs are assumed to be located in an internal squared network of length two which is located within the square network of length four. Furthermore, the PBS and the SAP are assumed to be located on side of the center of the network. The channel gains of link pairs among PUs, SUs, the PBS, and the SAP are independently generated from complex Gaussian distribution with zero mean and unit variance.

\subsection{Analysis of PU's average capacity}

Figure 3 shows the comparison result of the PU's average capacity achieved by different approaches, which include the optimal allocation, random matching, stable matching, swap matching and random matching with swap. As can be clearly seen, when $\gamma_{P U}=10 \mathrm{~dB}$ and $\gamma_{S U}=25 \mathrm{~dB}$, the PU's average capacity achieved by the optimal allocation approach is larger than the one achieved by other approaches. This is since that this approach is run by the exhausting search for solving the optimization problem. However, the time complexity of this approach is very high. Thus, we propose the stable matching and the swap matching approaches to make the PU's capacity achieve closer to the one of optimal allocation approach, but their time complexity are relatively low as compared to the optimal allocation one.

Figure 3 also shows that the PU's average capacity achieved by the swap matching approach is larger than the one achieved by the stable matching approach, and is also larger than the one achieved by the random matching approach. It can be clearly seen, the PU's capacity increases significantly with the stable matching and the swap matching as compared to the case of random matching.

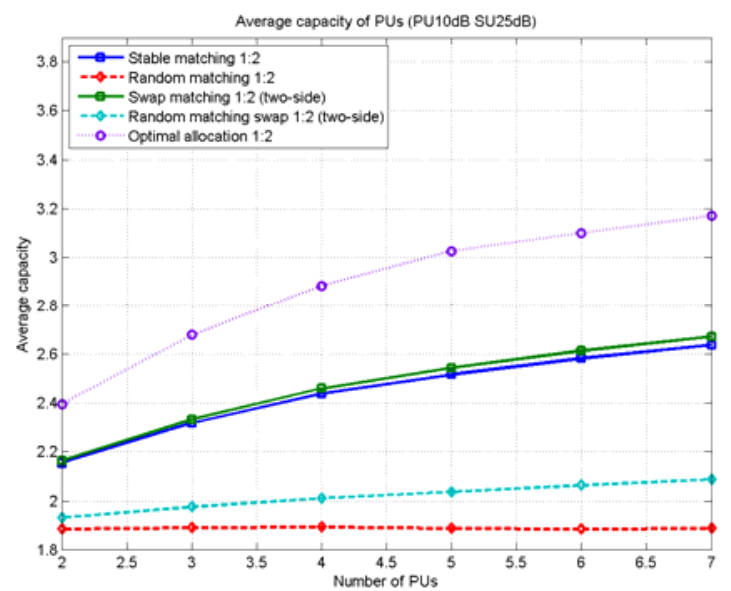

Figure 3 The average capacity of PU.

\subsection{Analysis of SU's capacity}

Fig. 4 shows the comparison result of the SU's average 
capacity achieved by different approaches, which include the optimal allocation, random matching, stable matching, swap matching and random matching with swap. We see that, the SU's average capacity achieved by the swap matching approach is larger than the one achieved by the stable and the random matching approaches.

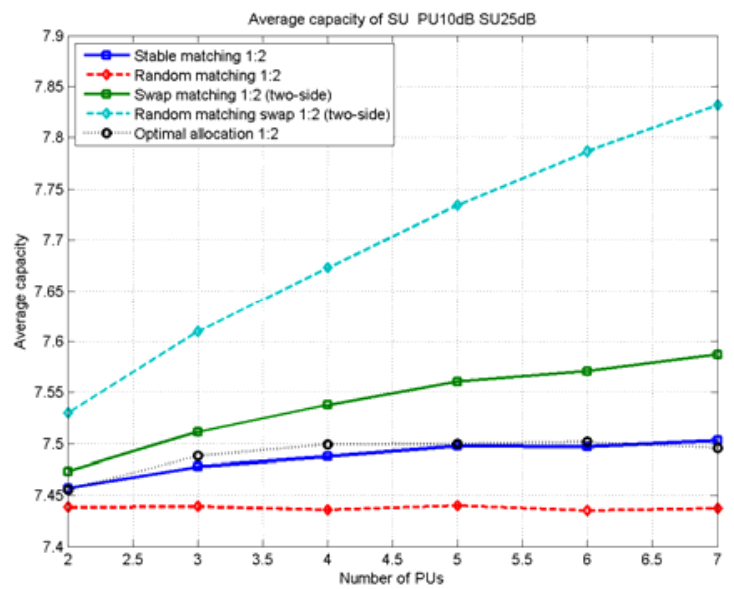

Figure 4 The average capacity of SU.

\section{Conclusions}

We have proposed a stable matching with peer effects approach to studying the hierarchical spectrum sharing strategies between multiple PUs and multiple SUs in a multichannel CCRN. The analytical arguments were verified by simulation, where the benefits of the stable matching and the swap matching approaches were visually presented for all PUs and SUs in the multi-channel CCRN.

\section{Conclusions}

This work was supported by the Ministry of Science and Technology of the Republic of China under Grant number 103-2221-E-005-028.

\section{References}

(1) O. Simenone, I. Stanojev, S. Savazzi, Y. Bar-Ness, U. Spagnolini and R. Pickholtz, "Spectrum leasing to cooperating secondary ad hoc networks," IEEE J. Select. Areas Commun., vol. 26, pp. 203-213, Jan.2008

(2) J. Zhang and Q. Zhang, "Stackelberg game for utilitybased cooperating cognitive radio networks," in Proc. ACM MobiHoc, pp. 23-31 May 2009
(3) X. Wang, Z. Li, P. Xu, Y. Xu, X. Gao, and H.-H Chen, "Spectrum sharing in cognitive radio networks - an auction-based approach," IEEE Trans Syst., Man, Cybern. B, vol. 40, pp. 587-596,Jun. 2010

(4) Y.-W. Chan, F.-T. Chien, R. Y. Chang, M-K. Chang, and Y-C. Chung, "Spectrum sharing in multi-channel cooperative cognitive radio networks: A coalitional game approach," Wireless Networks, vol. 19, pp. 15531562, Oct. 2013.

(5) D. Gale and L. S. Shapley, "College admissions and the stability of marriage," The American Mathematical Monthly, vol. 69,No. 1,pp. 9-15, 1962.

(6) K. Iwama and S. Miyazaki "A survey of the stable marriage problem and its variants," in IEEE ICKS 2008, 2008, pp. 131-136.

(7) H. Xu and B Li, "Seen as stable marriages." In Proc. IEEE INFOCOM'11, Shanghai, Apr. 2011, pp. 586-590

(8) E. A. Jorswieck, "Stable matchings for resource allocation in wireless networks." in IEEE Digital Signal Processing (DSP), 2011, pp. 1-8

(9) Y. Y. A. Leshem and E. Zehavi, "Stable matching for channel access control in cognitive radio system," in IEEE Cognitive Information Processing (CIP), 2010, pp. 470-475.

(10) S. Bayat, R. H. Y. Louie, L. Yonghui, and B. Vucetic, "Cognitive radio relay networks with multiple primary and secondary users: Distributed stable matching algorithms for spectrum access,' in Proc. IEEE ICC '11, Kyoto, Jun. pp. 1-6 Jun. 2011.

(11) A. Leshem, E. Zehavi, and Y. Yaffe, "Multichannel opportunistic carrier sensing for stable channel access control in cognitive radio systems," IEEE J. Select. Areas Commun., vol. 30, NO. 1, pp. 82-95, 2012

(12) E.-B. C. Anthony Chong and B. H. A. Wierman, "Peer effects and stability in matching market."

(13) A. E. Roth, "The evolution of the labor market for medical interns and residents: a case study in game theory," The Journal of Political Economy, pp. 991-1016, 1984

(14) I. Akyildiz, W. Lee, M. Vuran, and S. Mohanty, "Next generation/dynamic spectrum access/cognitive radio wireless networks: A survey," Elseiver Computer Networks, vol. 50, pp. 2127-2159, Sep. 2006

(15) S. Haykin, "Cognitive radio: brain-empowered wireless communication," IEEE J. Select. Areas Commun., vol. 23, pp. 201-220, Feb. 2005. 